\title{
LA INCIDENCIA DE LA INNOVACIÓN SOBRE LA CREACIÓN DE VALOR: PROPUESTA DE UN MODELO DESDE LA PERSPECTIVA FINANCIERA*
}

\author{
JORGE ALBERTO RIVERA GODOY** \\ UNIVERSIDAD DEL VALLE
}

Recibido/ Received/ Recebido: 13/01/2012 - Aceptado/ Accepted / Aprovado: 25/09/2012

\section{Resumen}

\begin{abstract}
Se presenta un modelo conceptual que permite medir y gestionar los efectos de la innovación sobre el valor de la empresa. Como marco teórico se realiza una descripción de la innovación como un inductor estratégico de la cadena de valor de la organización, y, siguiendo los preceptos de la gerencia del valor, se presenta los métodos para calcular el valor económico agregado de la empresa, el valor de mercado agregado y el valor de empresa, y su relación con la innovación. El modelo muestra las variables de entrada y salida, y los factores que hacen parte de su proceso, posteriormente se dimensiona el impacto sobre el valor económico agregado de cada una de los tipos de innovaciones mediante la formulación de un modelo de regresión lineal múltiple. El modelo se enfoca a la evaluación de la intensidad de las inversiones en diferentes actividades de innovación.
\end{abstract}

Palabras clave: Innovación, Cadena de valor, Creación de valor, Valor económico agregado (EVA).

\section{INCIDENCE OF INNOVATION ON CREATION OF VALUE: PROPOSAL OF A MODEL FROM THE FINANCIAL APPROACH}

\begin{abstract}
This paper presents a conceptual framework that allows measuring and managing the effects of innovation on the value of a company. The theoretical framework contains a description of innovation as a strategic inductor of company's chain of value, and it follows the fundaments of value management. The methods to calculate the economic value added of the company, the value of added market and company's value, and its relation with innovation. The model shows the in and out variables, and factors included in the process, then the impact on economic value added is dimensioned foreach one the innovation types, through a multiple linear regression model. The model emphasizes in the assessment of investment intensity in various activities of innovation.
\end{abstract}

Keywords: Innovation, Chain of value, Value creation, Economic value added.

* Este trabajo hace parte de uno de los desarrollos del proyecto de investigación "¿Es la innovación en gestión, fuente de valor económico para las empresas en Colombia?” del convenio 163 de 2007 entre Colciencias y el Instituto de Prospectiva, Innovación y Gestión del Conocimiento de la Universidad del Valle. Fecha de inicio: Agosto 2009.

** Profesor titular de la Universidad del Valle. Doctor Distinguido "Cum Laude" en Ciencias Económicas y Empresariales, Universidad Autónoma de Madrid, 1999. Especialista en Finanzas, Universidad del Valle, 1991. Contador Público, Universidad Santiago de Cali. Jefe del Departamento de contabilidad y finanzas de Univalle. Coordinador del Grupo de Investigación de Generación de Valor Económico (GIGVE) de Univalle. Correo electrónico: jorge.rivera@correounivalle.edu.co 


\title{
A INCIDÊNCIA DA INOVAÇÃO SOBRE A CRIAÇÃO DE VALOR: PROPOSTA DE UM MODELO DESDE A PERSPECTIVA FINANCEIRA
}

\section{Resumo}

\begin{abstract}
Apresenta-se um modelo conceitual que permita medir e gerenciar os efeitos da inovação sobre o valor da empresa. Como marco teórico se realiza uma descrição da inovação como um indutor estratégico da corrente de valor da organização, e, seguindo os preceitos da gerência de valor, se apresentam os métodos para calcular o valor econômico agregado da empresa, o valor de mercado agregado e o valor da empresa, e sua relação com a inovação. O modelo mostra as variáveis de entrada e saída, e os fatores que fazem parte de seu processo. Posteriormente se dimensiona o impacto sobre o valor econômico agregado de cada um dos tipos de inovações mediante a formulação de um modelo de regressão linear múltipla. O modelo se enfoca na avaliação da intensidade dos investimentos em diferentes atividades de inovação.
\end{abstract}

Palavras chave: Inovação, Corrente de valor, Criação de valor, Valor econômico agregado (EVA).

Rivera, J. (2012) La incidencia de la innovación sobre la creación de valor: propuesta de un modelo desde la perspectiva financiera. En: Revista de la Facultad de Ciencias Económicas de la Universidad Militar Nueva Granada. rev.fac.cienc.econ, XX (2).

JEL: O31, O32.

\section{Introducción}

El objetivo fundamental de las empresas es generar flujos de efectivo futuros que garanticen su sostenibilidad y crecimiento, en un medio de una alta competitividad y de un constante cambio en todos los órdenes en este nuevo siglo. Las innovaciones, como tales, son promotoras de cambios, y por eso son un componente estratégico de primer orden dentro de las organizaciones, al ser reconocidas como micro-inductores de valor económico. Sin embargo el verdadero efecto sobre el desempeño financiero de la empresa, no se hace tan evidente con la lectura de los indicadores tradicionales contables y del mercado ya que estos engloban resultados de actividades innovadoras y no innovadoras de las empresas. Entonces ¿cómo las empresas pueden conocer si la inversión en innovación le está generando los resultados económicos esperados?

Algunos investigadores han presentado modelos que evalúan la relación de la innovación con indicadores de desempeño (ej. crecimiento en ventas, rendimiento, valor de la firma, etc.), como se muestra en el apartado 4; pero sin enfatizar sobre las categorías de innovación, ni su condición de inductor de valor económico.

La forma cómo se puede determinar el impacto de las diferentes modalidades de innovación sobre el valor de la empresa, es una de las inquietudes que no se ha resuelto plenamente, por la complejidad de conocer y medir todos los factores influyentes. En este trabajo se pretende presentar un modelo que reúna algunos elementos más relevantes para evaluar el efecto de la innovación sobre la creación de valor económico siguiendo los lineamientos de la gestión basada en el valor.

Para tal propósito se ha estructurado este artículo en cinco apartados. En el primero se presenta una descripción de la innovación: su concepto, principios y tipos, como, también, su objetivo financiero; en el segundo se define y se formula la medición del valor económico agregado, el valor de mercado agregado y el valor de empresa; en el tercero se trata la innovación como una competencia esencial en la cadena de valor; en el cuarto se propone un modelo conceptual que permita interrelacionar las decisiones 
de innovar con los resultados de valor económico y, finalmente, en el quinto, las conclusiones.

El diseño de este modelo de gestión financiera contribuye a la academia a reflexionar sobre la información utilizada para evaluar el desempeño de organizaciones innovadoras: ¿Cuáles son las limitaciones de nuestro sistema de información contable?, ¿cuáles son las restricciones de la información del mercado?, ¿se requiere ser procesada a través de modelos? ¿es un problema interdisciplinario, donde la gestión del valor y la econometría pueden aportar?; como, también, poner a consideración el diseño conceptual del modelo: ¿cuáles son sus beneficios?, ¿cuáles sus limitaciones?, ¿en qué se puede mejorar?, y al realizar su contraste empírico: ¿qué tan confiable es?, y si ¿cumple su objetivo?

\section{Caracterización y propósito financiero de la innovación}

Aunque existen diferentes conceptos de innovación, una de las más aceptadas por la comunidad científica es la expresada en Manual de Oslo que la define como "la introducción de un nuevo, o significativamente mejorado, producto (bien o servicio), de un proceso, de un nuevo método de comercialización o de un nuevo método organizativo, en las prácticas internas de la empresa, la organización del lugar de trabajo o las relaciones exteriores" (ODCE \& Eurostat, 2006: 56).

Tabla 1. Principios de la innovación ${ }^{1}$

\begin{tabular}{|l|l|}
\hline \multicolumn{1}{|c|}{ Se debe hacer (reglas) } & No se debe hacer (prohibiciones) \\
\hline - Comenzar con análisis de las & - Innovaciones que requiera mu- \\
fuentes de oportunidades. & cha astucia para su manejo o \\
- Observar, preguntar y escuchar a & producción. \\
la gente. & Diversificar: La innovación no \\
- Tener una aplicación simple y & debe cumplir con varias funcio- \\
específica. & nes. \\
- Iniciar a pequeña escala. & - Innovar para el futuro, se debe \\
- Ser líder en su campo. & hacer para el presente. \\
\hline Condiciones: & \multicolumn{2}{|l}{ - Se requiere mucho trabajo, diligencia, constancia y dedicación. } \\
- Estar siempre cerca, enfocada y dirigida al mercado. \\
\hline
\end{tabular}

Drucker (1988) señala que la gran mayoría de innovaciones efectivas han sido el resultado de una actividad sistemática, intencional y organizada, más que una derivación de una" idea brillante". Establece como principios para su éxito, cinco reglas, tres prohibiciones y dos condiciones, como se muestra en la Tabla 1.

ODCE y Eurostat $(2006,58-60,62)$ clasifican y definen cuatro tipos de innovaciones:

- Innovación de producto: "corresponde a la introducción de un bien o de un servicio nuevo, o significativamente mejorado, en cuanto a sus características o en cuanto al uso al que se destina. Se incluye la mejora significativa de las características técnicas, de los componentes y los materiales, de la informática integrada, de la facilidad de uso u otras características funcionales".

- Innovación de proceso: "es la introducción de un nuevo, o significativamente mejorado, proceso de producción o de distribución. Ello implica cambios significativos en las técnicas, los materiales y los programas informáticos".

- Innovación de mercadotecnia: "es la aplicación de un nuevo método de comercialización que implique cambios significativos del diseño o el envasado de un producto, su posicionamiento, su promoción o su tarificación".

- Innovación de organización: "es la introducción de un nuevo método organizativo en las prácticas, la organización del lugar de trabajo o las relaciones exteriores de la empresa".

En el medio empresarial, como académico, se ha considerado la innovación como una estrategia que permite impulsar el crecimiento y rendimiento financiero, y ser un factor determinante en la consecución de utilidades económicas (Sorescu \& Spanjol, 2008). Aunque se espera que los proyectos innovadores generen un valor actual neto positivo más alto, por el mayor riesgo que se asume, en comparación con los proyectos comunes, que redunden en mayor valor de la empresa, los resultados de investigaciones han tenido conclusiones diferentes.

1 Fuente: Elaboración propia con base en Drucker (1988, 162-166). 
Mientras que algunos estudios han encontrado una relación positiva entre la innovación y el valor de la empresa (Koski, \& Kretschmer, 2010; Pauwels, Silva-Risso, Srinivasan \& Hanssens, 2004 y Sorescu, Chandy \& Prabhu, 2003), otros encuentran que esta relación es mínima, o no existe (Chaney, Devinney \& Winer, 1991; Geroski, Machin \& Van Reenen, 1993 y Foster \& Kaplan, 2001). Lo que muestra la necesidad de diseñar modelos que permitan medir y administrar la cadena de valor que genera la innovación.

\section{EVA, VMA y valor de empresa}

En el medio académico y empresarial se viene considerando últimamente la gestión basada en el valor como un sistema que permite medir y evaluar el desempeño financiero de organizaciones, que presenta algunas ventajas sobre los tradicionales análisis de datos contables y de mercado (Martin \& Petty, 2001). Una de las metodologías más conocida de la gestión basada en el valor es el valor económico agregado (EVA), con el que se pretende administrar la creación de valor mediante la medición, evaluación y control de la utilidad residual.

Stewart $(2000,163)$ propone calcular el EVA como la diferencia entre la tasa de rendimiento del activo, UODI / ANO, y el coste del capital, Ko, y luego multiplicarlo por el activo neto operacional del negocio, ANO; así:

$E V A=(U O D I / A N O-K o)(A N O)$

Donde, UODI es la utilidad operacional después de impuestos.

También Stewart propone hallarlo de una segunda manera, que consiste en multiplicar los dos componentes del porcentaje de utilidad o pérdida residual (UODI /ANO - Ko), por el activo neto operacional, quedando EVA igual a:

$E V A=(U O D I / A N O)(A N O)-(K o)(A N O)$; simplificando se tiene:

$E V A=U O D I-(A N O)(K o)$
El costo medio ponderado de capital, Ko, es el resultado de combinar el costo de la deuda Ki y el costo del capital propio Ke. Según Miller \& Modigliani (1963, 441) el Ko es igual a:

$K o=K e(1-L)+K i(1-t) L$

Donde, $L$ es el coeficiente de endeudamiento, Ki (1 - $t$ ) es el costo después de impuestos de la deuda, y $t$ la tasa de impuesto.

Como en las finanzas modernas, el concepto de valor de empresa está ligado a la capacidad de generar utilidades económicas en el futuro, y no a un único periodo como sucede con el EVA, se hace necesario complementar esta medida con el valor de mercado agregado, VMA, que tradicionalmente se conoce como la prima que el mercado concede a la companía en relación con el capital colocado por los accionistas, pero que es equivalente al valor actual de las utilidades residuales proyectadas, es decir:

$\mathrm{VMA}=\sum_{t=1}^{t=n}\left(\right.$ Utilidades residuales) ${ }_{t} /\left(1+K o_{t}\right)^{t}$

Una forma de calcular las utilidades residuales es por medio de los EVA, por lo que:

$V M A=\sum_{t=1}^{t=n} E V A_{t} /\left(1+K o_{t}\right)^{t}$

Conociendo que el valor de mercado agregado es igual a:

$V M A=$ Vr. mercado de las acciones en Bolsa VM (-) Vr. invertido por inversionistas $A N O_{t-1}$

Se determina el valor de mercado de la empresa, VM, como:

$\mathrm{VM}=\mathrm{ANO}_{\mathrm{t}-1}+\mathrm{VMA}$

O de otra forma:

$\mathrm{VM}=\mathrm{ANO}_{\mathrm{t}-1}+\sum_{\mathrm{t}=1}^{\mathrm{t}=\mathrm{n}} E V A_{\mathrm{t}} /\left(1+\mathrm{Ko}_{\mathrm{t}}\right)^{\mathrm{t}}$ 
Aunque algunos estudios cuestionan el EVA como medida de creación de valor por la escasa correlación entre el EVA y la creación de valor para los accionistas (Fernández \& Reinoso, 2003), o por la escasa relación entre el EVA y el VMA (Lovata, 2001), o porque existe una mayor correlación entre el incremento anual del VMA y la UODI que la que hay entre el aumento del VMA y el EVA (Fernández, 2001); otros resultados muestran lo contrario: altas y positivas correlaciones entre EVA y VMA (Iñiguez \& Poveda, 2001; Hall \& Brummer, 1999), mayor correlación entre el EVA y la rentabilidad, que la que hay entre esta última y las medidas tradicionales contables (O’Byrne, 1996; Lehn \& Makhija, 1997).

Para este estudio se reconoce el EVA no solo por las evidencias de los estudios citados, sino por permitir evaluar el desempeño financiero de las empresas en medios con limitado avance en el desarrollo de mercados de capitales y con escasa información de mercado disponible.

\section{La innovación como competencia esencial en la cadena de valor}

Cuando las empresas buscan crear valor, deben establecer una estrategia para cada unidad de negocio, donde se identifique su competencia esencial, con el propósito de lograr una ventaja competitiva sostenible en el tiempo, lo que daría mayores posibilidades de lograr rentabilidades superiores a sus competidores.

La competencia esencial genera una ventaja competitiva con mayor duración en la medida que logre mantener las siguientes cualidades: i) ser mejor que la competencia; ii) ser valiosa: ésta se puede lograr por su diferenciación o ventaja en costos, como lo plantea Porter (1988); iii) ser rara; y iv) ser difícil de copiar, y que pueda ser aplicable a clientes o mercados o productos (García, 2003, 40-42).

Una de las principales fuentes de competencias esenciales es la innovación, cuyo ciclo de vida útil es cada vez menor dado el crecimiento de la competencia internacional y de los constantes cambios en las condiciones del mercado. Esto significa que hoy en día el tiempo para que una nueva innovación pueda amortizar los costos de inversión y generar utilidad económica a los propietarios, se ha reducido.

Pero las enormes inversiones que deben ser destinadas a procesos de innovación, han hecho que las empresas tengan como estrategia no solo responsabilizar al departamento de $\mathrm{I}+\mathrm{D}$, sino a todas las áreas funcionales de la empresa, puesto que estas innovaciones se pueden presentar en cada una de las etapas de la cadena de valor de la empresa. Cada una de las etapas de la cadena de valor requiere de una estrategia que impulse la generación de valor.

En la Ilustración 1 se esquematiza como ejemplo, una cadena de valor de una empresa manufacturera, donde se separan las actividades primarias y de apoyo, y sus componentes estratégicos.

Cualquiera de estas competencias fundamentales puede haber surgido de una innovación. Por lo tanto, solo las innovaciones con ventaja competitiva asegurarán ser impulsoras de valor.

En la Ilustración 1 se puede observar que aunque existe un componente estratégico de innovación, que podría estar liderado por una unidad organizacional, no es la única fuente de innovación, sino que ésta puede surgir de cualquier otro componente estratégico de la cadena de valor.

Dentro de la cadena de valor es importante definir, clasificar y medir los inductores de valor. Los inductores de valor son aquellos agentes impulsores de valor de la empresa, que de acuerdo a su naturaleza pueden clasificarse en:

- Micro-inductores, muchos de los cuales pueden ser originados de innovaciones.

- Inductores estratégicos, como las competencias esenciales que tienen como fuente las innovaciones, y que potencialmente son ventajas competitivas. También los proyectos de innovación que ofrezcan rendimientos de capital superiores a la tasa mínima de rendimiento, son impulsores estratégicos de valor. 
Ilustración 1. La innovación y la cadena de valor²

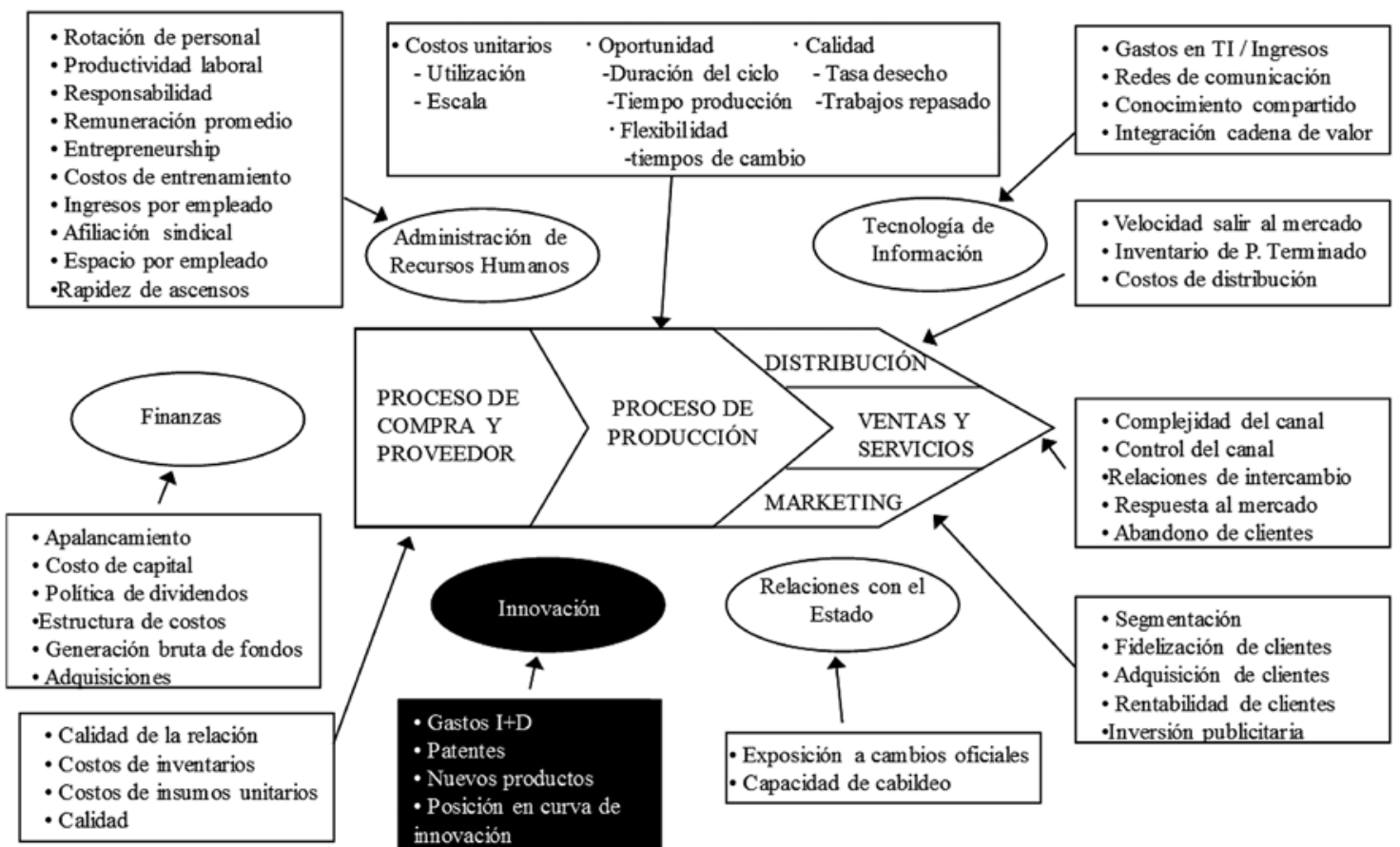

- Inductores operativos y financieros que se apoyan de los inductores estratégicos y muestran la eficiencia (rotación de activos) y la eficacia (margen de utilidad) de toda la cadena de valor. El apalancamiento es un inductor financiero que revela la competencia financiera de la empresa, para que sus accionistas se beneficien de un apalancamiento financiero positivo.

- Macro-inductores que recopilan el efecto de los propulsores de valor micros, estratégicos, operativos y financieros. En un primer nivel, el rendimiento sobre activos (efectividad), refleja la combinación de la eficiencia y eficacia, y el costo de capital es el resultado de una estructura de capital óptima. En segundo nivel el EVA, que muestra el valor económico creado en un período, que en condiciones óptimas debería ser promovido por un ANO bajo, una UODI alta y un Ko bajo. En un tercer nivel el VMA que muestra el valor actual de EVAs proyectados en

el futuro, conforme al tiempo estimado del ciclo de vida la innovación; y el último nivel el valor de la empresa que surge del VMA más la inversión inicial (Ilustración 2).

\section{Propuesta de un modelo conceptual}

En el diseño de la propuesta del modelo conceptual se consultaron algunos estudios que investigan, mediante la aplicación de un modelo, el efecto económico de la innovación. Dentro de ellos cabe destacar los que de una manera más directa sirvieron de fuente de inspiración para el diseñado en este estudio:

- Sorescu \& Spanjol (2008) formulan un modelo para examinar el efecto del incremento y progreso de la innovación sobre el valor de la firma y el riesgo, presentando como principales ecuaciones las determinantes de la $\mathrm{Q}$ de Tobin, de los rendimientos anormales y del riesgo. 
- Coad \& Rao (2007) plantean un modelo de panel autoregresivo para observar la evolución del crecimiento de las ventas, el empleo las ganancias con relación al crecimiento de los gastos de investigación y desarrollo.

- House, Parks \& Lindstrom (1994) diseñan un modelo que permita relacionar la rentabilidad y los gastos de investigación y desarrollo con simulaciones entre dos empresas y dos industrias.

- Morbey \& Reither (1990) con un modelo de correlación evalúan si hay relación directa entre el gasto de investigación y desarrollo y la rentabilidad.

- Grabowski \& Mueller (1978) mediante un análisis de corte transversal, plantean un modelo para examinar la relación entre el rendimiento (como variable independiente) y la inversión en $\mathrm{I}+\mathrm{D}$, la concentración del mercado, el tamaño de la firma y el crecimiento de la industria (como variables dependientes).

A continuación se desarrolla el modelo propuesto:

\subsection{Objetivo y alcance}

Se busca presentar un modelo conceptual que permita medir y gestionar los efectos de la innovación sobre el valor de la empresa, haciendo un seguimiento a los principales inductores de valor bajo un enfoque sistémico de la gerencia basada en el valor. El modelo parte de dos ejes: la intensidad de la innovación y los efectos. En el primero se muestra la cantidad de inversión en cada uno los tipos de innovación (sea gasto, costo o capitalización en activo) y en el origen de los fondos, que genera un costo de capital. En el

Ilustración 2. Inductores de valor ${ }^{3}$

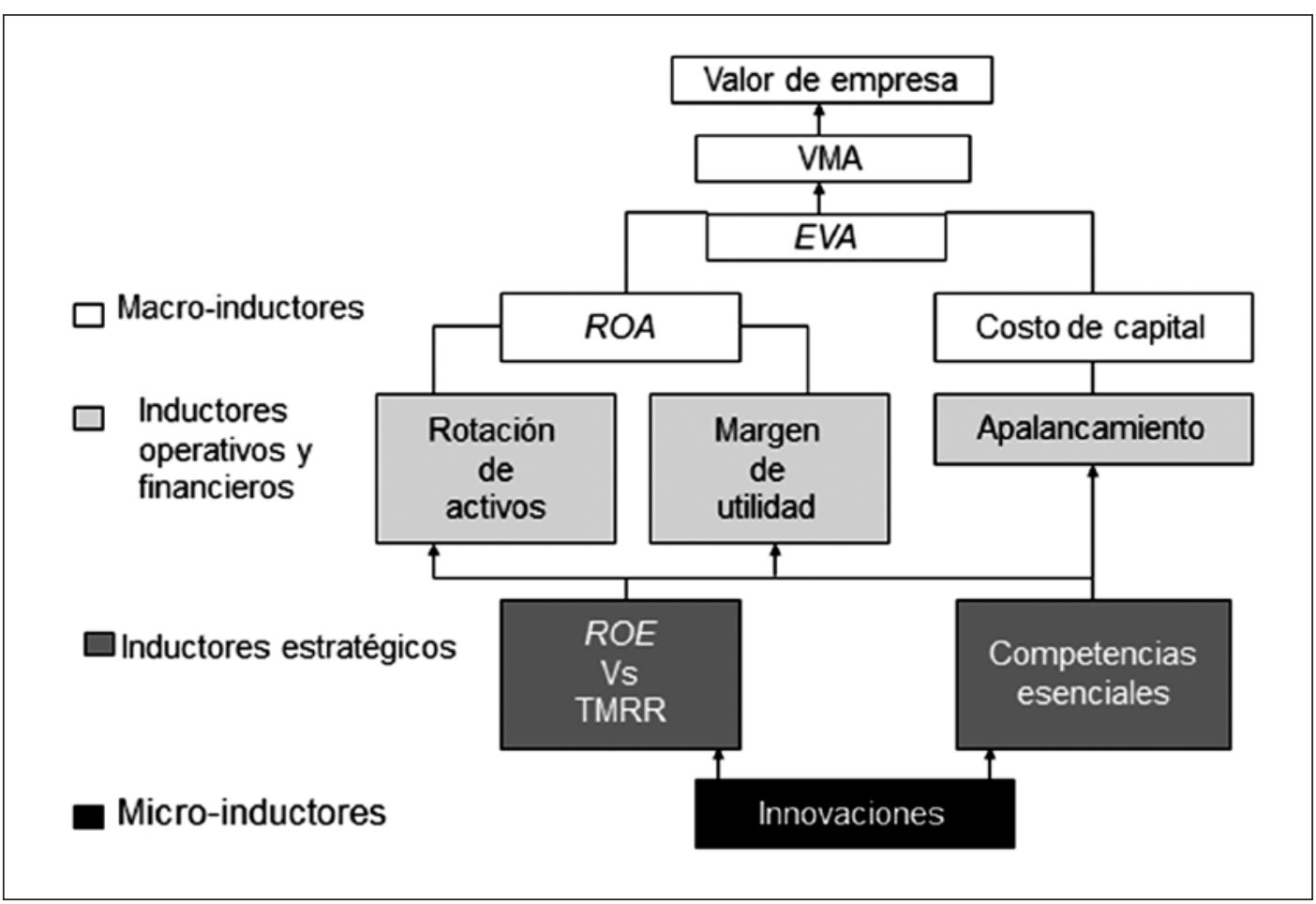

3 Fuente: Elaboración propia. 
segundo el efecto sobre los inductores que explican la cadena de valor económico de la empresa. Los efectos permitirán evaluar la toma de decisiones de nuevas innovaciones (Ilustración 3).

El modelo presenta como variables de input la información de la intensidad por cada una de las actividades de innovación y los costos por las fuentes de uso utilizadas; que va a generar dos variables output: la primera muestra los efectos económicos a través de indicadores de gestión de valor, y la segunda, los efectos de la intensidad de cada actividad de innovación sobre el EVA, como se observa en la Ilustración 4.

\subsection{Definición y medición de variables input}

En el Manual de Oslo se clasifican como actividades innovadoras, la investigación y desarrollo experimental, las actividades de innovación en producto y proceso, y las actividades innovadoras en mercadotecnia y organización (ODCE \& Eurostat, 2006), cuya intensidad en el costo y gasto se identifica así:

Cid $=$ costo y gasto de investigación y desarrollo experimental.

$C s=$ costo y gasto de investigación de innovación en producto.

$C p=$ costo y gasto de investigación de innovación en proceso.

$\mathrm{Cm}=$ costo y gasto de investigación de innovación en mercadotecnia.

Co $=$ costo y gasto de investigación en innovación en organización.

La intensidad en la capitalización de la inversión de cada una de las actividades se expresa así:

Iid = Activos netos operacionales asignados a innovación y desarrollo.

Is = Activos netos operacionales asignados a innovación en producto.
Ip = Activos netos operacionales asignados a innovación en proceso.

Im = Activos netos operacionales asignados a innovación en mercadotecnia.

Io = Activos netos operacionales asignados a innovación en organización.

Los recursos financieros necesarios para la innovación pueden ser por recursos de capital propios o deuda, cada uno de ellos tiene un costo. El costo del capital propio, Ke, más el costo de la deuda después de impuestos, $K i(1-t)$, genera un costo de capital, Ko.

\subsection{Definición y medición de variables output}

Para las variables output 1 los indicadores financieros fundamentales ROA, costo de capital, EVA, VMA $y$ valor de empresa se presentaron sus definiciones $y$ mediciones en sección 3. Dado que el valor de empresa va a depender de los valores proyectados del EVA en un espacio de tiempo donde se estime que cumple la vida útil la innovación; se hace necesario conocer cuáles son aquellas actividades de innovación que más van a incidir sobre EVA, lo que para el modelo son las variables output 2 .

Si se parte de que para un periodo $t: E V A_{t}=\left(U O D I_{t}\right)$ - $\left(A N O_{t}\right)\left(K_{t}\right) ; y$ si se divide ambos términos por el $A N O_{t}$, se tendría:

$E V A_{t} / A N O_{t}=(U O D I) / A N O_{t}-\left(K o_{t}\right)$

Lo que significa que el valor agregado que genera una unidad monetaria del activo neto operacional sería equivalente a la diferencia entre rendimiento del activo neto operacional $R O A_{\mathrm{t}}$ y el costo de capital $\mathrm{Ko}_{\mathrm{t}}$ así:

$E V A_{t} / \mathrm{ANO}_{t}=R O A_{t}-K o_{t}$

Con lo que se puede asumir que el que el valor agregado que genera una inversión está en función del rendimiento del capital y del costo de capital: $E V A_{t} /$ $A N O_{t}=f\left(R O A_{t}, K o_{t}\right)$.

4 Fuente: Elaboración propia. 
Ilustración 3. Mapa conceptual del efecto de la innovación sobre el valor de empresa ${ }^{4}$

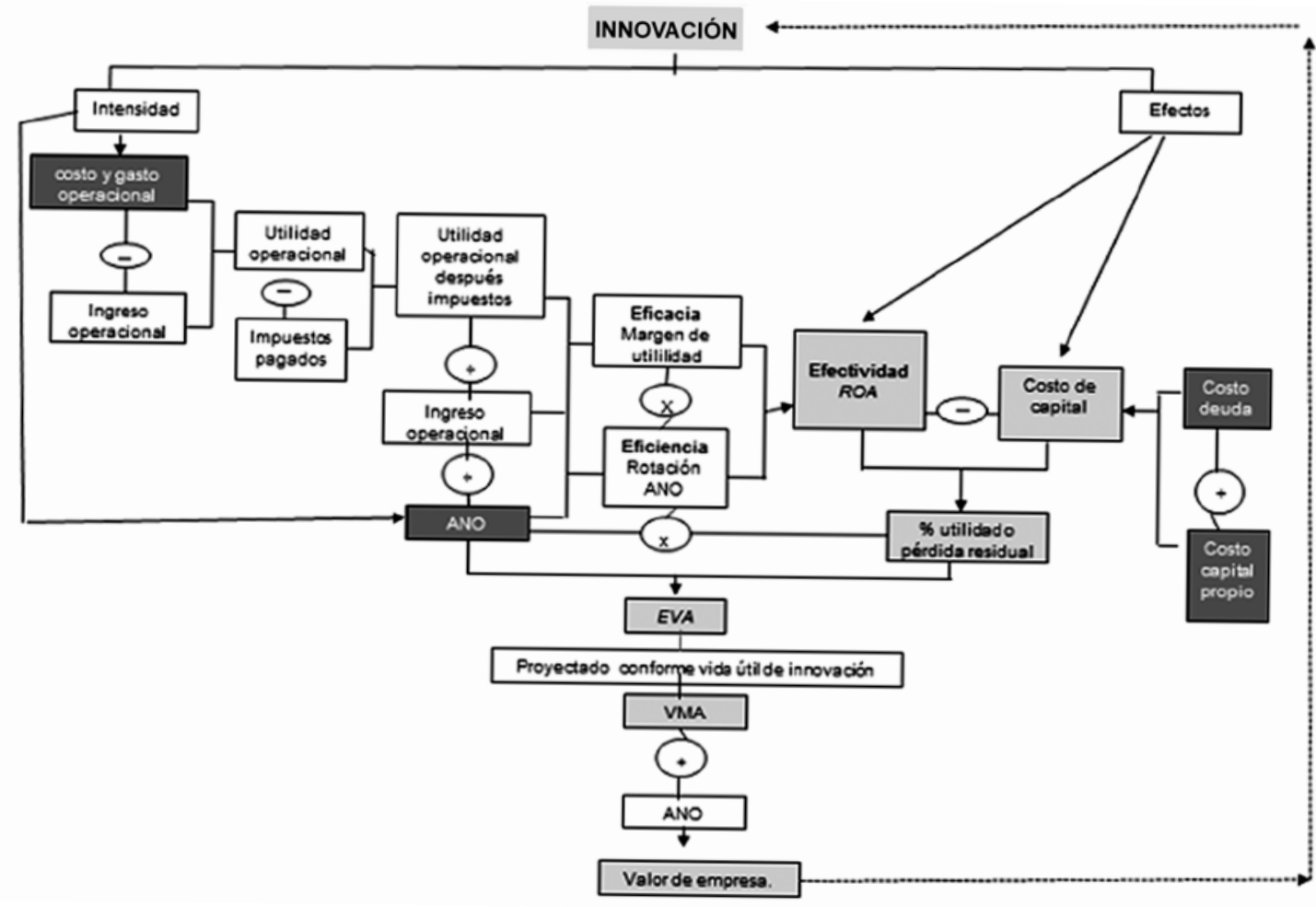

Ilustración 4. Variables de entrada y salida del modelo 5

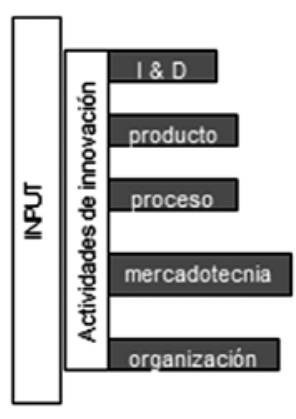

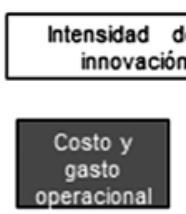

Fuente de recursos

Costo deuda
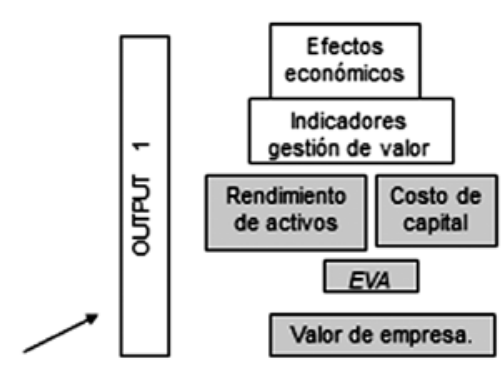

EVA

Valor de empresa.

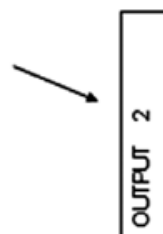

Efectos de la intensidad de cada actividad de innovación sobre EVA

Modelo econométrico

Betas $\mathrm{Y}=\mathrm{ROA}$ $\mathrm{Y}=\mathrm{Ko}_{\mathrm{O}}$ $Y=E V A$

5 Fuente: Elaboración propia. 


\subsubsection{Análisis de ROA}

Si se analiza primero $\mathrm{ROA}_{t}$ se tiene:

$R O A_{t}=\left[\right.$ Ingreso operacional $\left.-C_{t}-\mathrm{CI}_{t}\right] / \mathrm{ANO}_{t}$

Donde:

$\mathrm{CN}_{t}=$ Costos y gastos operacionales por actividades normales.

$C I_{t}=$ Costos y gastos operacionales por actividades que involucren innovación, y será equivalente a: $\mathrm{CI}_{t}$ $=C i d_{t}+C s_{t}+C p_{t}+C m_{t}+C o_{t}$

$A N O_{t}=I n_{t}+I i d_{t}+I s_{t}+I p_{t}+I m_{t}+I o_{t}$, donde $I n_{t}$ son los activos netos operacionales para actividades normales.

De tal forma se puede replantear el rendimiento de los activos netos operacionales así:

$R O A_{t}=$ [Ingreso operacional $-C_{t} N_{t}-C i d_{t}+C s_{t}+C p_{t}$ $\left.+C m_{t}+C o_{t}\right] /\left[\operatorname{In}_{t}+\operatorname{Iid}_{t}+\mathrm{Is}_{t}+\operatorname{Ip}_{t}+\operatorname{Im}_{t}+I o_{t}\right]$

Es decir, que el $R O A_{t}$ está determinado por algunos factores que muestran la intensidad de inversión en actividades de innovación. Por lo tanto se puede expresar matemáticamente así:

$R O A_{t}=f\left[\operatorname{Iid}_{t} / \mathrm{ANO}_{t} ; \mathrm{Is}_{t} / \mathrm{ANO}_{t} ; \mathrm{Ip}_{t} / \mathrm{ANO}_{t} ; \mathrm{Im}_{t} / \mathrm{ANO}\right.$; $\left.\mathrm{Io}_{t} / \mathrm{ANO}_{\mathrm{t}}\right]$

Esta incidencia se puede determinar con un modelo de regresión lineal múltiple según ecuación (13).

$R O A_{t}=\alpha+\beta_{1}\left(\operatorname{Iid}_{t} /\right.$ ANO $\left._{t}\right)+\beta_{2}\left(I_{t} / \mathrm{ANO}_{t}\right)+\beta_{3}\left(\mathrm{Ip}_{t} /\right.$ $\left.A N O_{t}\right)+\beta_{4}\left(\operatorname{Im}_{t} / A N O_{t}\right)+\beta_{5}\left(I_{t} / A N O_{t}\right)+u_{t}$

Donde:

$\alpha=$ efecto constante de la inversión de cada actividad innovadora.

$\beta=$ vector de la inversión de cada actividad innovadora sobre la rentabilidad de los activos netos operacionales; el signo + ó -, indicará si el impacto es positivo o negativo, y solo se debe considerar si estadísticamente es significativo.

$u_{\mathrm{t}}=$ es el error aleatorio.

\subsubsection{Análisis de Ko}

Si se analiza Ko se tiene:

El costo de capital de los fondos de recursos destinados para todas actividades de la empresa es igual a:

$K o_{t}=K i_{t}\left(1-t_{t}\right) L_{t}+K e_{t}\left(1-L_{t}\right)$

Siguiendo esta formula matemática se puede hallar el costo de capital para los recursos financieros desti-

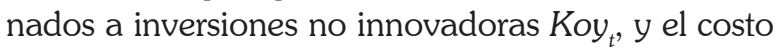
de capital para las fuentes de capital asignadas a inversiones innovadoras Kox.

Como el total de recursos utilizados por la empresa para todas las actividades es $A N O_{t}$, la ponderación destinada a actividades no innovadoras es igual a $\left(\mathrm{In}_{t} / \mathrm{ANO}_{\mathrm{t}}\right)$, y la destinada a actividades innovadoras equivalente a $\left(\mathrm{ANO}_{t}-\mathrm{In}_{t}\right) / \mathrm{ANO}_{t}$

De esta forma se podría formular el costo de capital así:

$K o_{t}=\left(\operatorname{In}_{t} / A N O_{t}\right) \operatorname{Koy}_{t}+\left[\left(\mathrm{ANO}_{t}-\mathrm{In}_{t}\right) / \mathrm{ANO}_{t}\right] \operatorname{Kox}_{t}$

Por lo que se puede afirmar que el Ko está determinado por algunos factores que muestran la intensidad de inversión en actividades de innovación; por lo tanto se puede expresar matemáticamente así:

$\mathrm{Ko}_{t}=f\left[\mathrm{Iid}_{t} / \mathrm{ANO}_{t} ; \mathrm{Is}_{t} / \mathrm{ANO}_{t} ; \mathrm{Ip}_{t} / \mathrm{ANO}_{t} ; \mathrm{Im}_{t} / \mathrm{ANO}\right.$ $\left.; \mathrm{Io}_{t} / \mathrm{ANO}_{t}\right]$

Y su efecto se puede comprobar con un modelo de regresión lineal múltiple según ecuación (17).

$K o_{t}=\alpha+\beta_{1}\left(\operatorname{Iid}_{t} /\right.$ ANO $\left._{t}\right)+\beta_{2}\left(I_{t} /\right.$ ANO $)+\beta_{3}\left(I_{t} /\right.$ $\left.A N O_{t}\right)+\beta_{4}\left(\operatorname{Im}_{t} /\right.$ ANO $\left._{t}\right)+\beta_{5}\left(I_{t} /\right.$ ANO $\left._{t}\right)+u_{t}$ 
Donde:

$\alpha=$ efecto constante de la inversión de cada actividad innovadora.

$\beta=$ vector de la inversión de cada actividad innovadora sobre el costo de capital. Si se tiene un signo positivo indica que la inversión de esta actividad de innovación tiene un impacto positivo sobre $\mathrm{Ko}_{t}$, caso contrario si es negativo; esto es válido siempre y cuando estadísticamente sea significativo.

El conocer el efecto de las inversiones en cualquiera de las cinco actividades innovadoras sobre el rendimiento de capital y costo de capital permitirá conocer cuáles son las inversiones innovadoras que más explican el valor económico agregado de la empresa.

\subsection{Operatividad del modelo}

Cuando la empresa está evaluando la posibilidad de realizar inversiones en innovación, se capturará la información requerida para determinar el $R O A_{t}$ y el $K o_{t}$ de los períodos $t$ proyectado como ciclo de vida de la innovación; con estos resultados se determinará:

El valor económico agregado $=E V A_{t}=R O A_{t}-$ $\mathrm{ANO}_{\mathrm{t}} \mathrm{Ko}_{\mathrm{t}}$

Posteriormente el valor de mercado agregado, como el valor presente de los $E V A_{\mathrm{t}}$ proyectados de $t=1$ hasta $t=n$ de la forma en que esta expresada la ecuación (5).

El valor de empresa, como: $V M=A N O_{t-1}+V M A$, o más detallada, como:

$\mathrm{VM}=\mathrm{ANO}_{\mathrm{t}-1}+\sum_{\mathrm{t}=1}^{\mathrm{t}=}\left[R O A_{\mathrm{t}}-\mathrm{ANO}_{\mathrm{t}} \mathrm{Ko}_{\mathrm{t}}\right] /\left(1+\mathrm{Ko}_{\mathrm{j}}\right)^{\mathrm{t}}$

Estos primeros resultados permitirán realizar comparaciones con los indicadores del sector, con las metas propuestas en el plan estratégico de la empresa y con el registro histórico de indicadores.
Posteriormente, se hará un análisis del efecto que tiene la inversión de cada una de las actividades innovadoras sobre el $R O A_{t}$ y $K_{o_{t}}$ con el análisis de los resultados de los dos modelos de regresión múltiple.

Con la evaluación de los efectos económicos y de la intensidad de las actividades de innovación sobre el EVA, el VMA y VM, los directivos podrán saber si las propuestas innovadoras satisfacen el propósito financiero de la empresa.

\subsection{Limitaciones y restricciones del modelo}

El modelo propuesto puede presentar hipotéticamente limitaciones de orden teórico una vez se cuente con datos para calcular la regresión múltiple, principalmente limitaciones tales como multicolinealidad y endogeneidad de las variables. Como la meta es sólo predecir el valor de la empresa con base en la intensidad de la inversión en actividades de innovación, la multicolinealidad no es un problema y el coeficiente de correlación cuadrático sigue cuantificando qué tan bueno es el modelo. Sin embargo, los valores $p$ específicos de las variables pueden prestarse a malinterpretación. La multicolinealidad ocurre cuando dos variables están relacionadas, es decir, cuando en esencia miden lo mismo. Una estrategia a adoptar es eliminar las variables que parezcan redundantes por estar correlacionadas.

La endogeneidad, por su parte, es un poco más difícil de tratar, ya que puede producir parámetros sesgados e inconsistentes. La solución natural más sencilla si hay una variable dependiente potencialmente endógena es reemplazarla por una variable "proxy", siendo usual utilizar la misma variable pero rezagada. Otra forma de solucionar un problema potencial de endogeneidad es usar técnicas de variables instrumentales, lo que permite a la vez medir la idoneidad de los instrumentos y el grado de endogeneidad. Un instrumento es una variable exógena que está fuertemente correlacionada con la variable potencialmente endógena y que influye a la variable dependiente solamente a través de la variable independiente potencialmente endógena. El estimador más común al que podría recurrirse en caso de endogeneidad es 
el estimador de mínimos cuadrados de dos etapas (Green, 2011).

Dado que los dos principales problemas hipotéticos que pueden surgir durante la aplicación del modelo tienen una solución, es de esperarse que estos problemas no constrinan la potencia teórica del modelo general, incluso a pesar de que este modelo se enfoque solamente en la intensidad de las inversiones en diferentes actividades de innovación; en una posible extensión del modelo podrían incluirse otros determinantes de carácter endógeno como el tamaño, o de carácter exógeno como el crecimiento del sector y la concentración del mercado, u otros como los enunciados en Langebaek \& Vásquez (2007, 9-15).

También debe tenerse en cuenta que el sistema de información contable tradicional no está adaptado para emitir datos de actividades innovadoras dentro de las empresas, lo que implica realizar ajustes en la clasificación de cuentas de costos, gastos y ANO en lo que corresponde a las cinco actividades innovadoras propuestas en el modelo.

\section{Conclusiones}

Es importante para las organizaciones conocer el efecto financiero de sus decisiones de invertir recursos en actividades innovadoras. Los métodos tradicionales de evaluación de desempeño de las empresas deben ajustarse a algunos modelos que le permita determinar si el objetivo financiero fundamental de crear valor se está cumpliendo.

En este artículo se tiene como objetivo poner a disposición de la comunidad académica un modelo conceptual que permite reconocer el enlace entre innovación y valor de empresa, mediante la combinación de una red de indicadores, basados en conceptos de la gerencia del valor, y un modelo de regresión múltiple para determinar el efecto de la inversión de diferentes tipos de innovación sobre el rendimiento del activo neto operacional y el costo de capital; principales inductores del valor económico agregado.

Al aplicar el modelo se puede presentar algunas restricciones con relación a la captura de la información de la inversión de las cinco actividades innovadoras, y multicolinealidad y endogeneidad de variables de la regresión múltiple; no obstante, la reclasificación de las cuentas por cada una de las categorías de innovación, la eliminación de variables redundantes, y el reemplazo por variable "proxy", son maneras de diluirlas, que deberían ser tenidas en cuenta al momento de emplearlo.

Cabe resaltar que el modelo se enfoca a la evaluación de la intensidad de las inversiones en diferentes actividades de innovación, pero es posible extenderlo, incluyendo otros determinantes como el tamaño de la empresa, el crecimiento del sector y la concentración del mercado, etc., dando lugar a la apertura de una línea de investigación en la creación y contrastes de modelos que permitan calcular y direccionar los efectos de la innovación sobre el valor de la empresa.

\section{Referencias}

Chaney, P., Devinney, T. \& Winer, R. (1991). "The impact of new product introductions on the market value of firms". En: Journal of Business, 64 (4): 573-610.

Coad, A. \& Rao, R. (2007). "Firm growth and R\&D expenditure". Max Planck Institute of Economics Evolutionary Economics Group: Papers on Economics and Evolution, 0710: 1 - 31.

Drucker, P. (1988). La innovación y el empresario innovador: la práctica y los principios. México: Editorial Hermes. (Original en inglés, 1985).

Fernández, P. (2001). Valoración de empresas (2a.ed.). Barcelona: Gestión 2000 S.A.

Fernández, P. \& Reinoso, L. (2003). "Shareholder value creators and shareholder value destroyers in USA. Year 2002". IESE Business School- Universidad de Navarra. Working paper 501.

Foster, R. \& Kaplan, S. (2001). Creative Destruction: Why Companies That Are Built to Last Underperform the Market-and How to Successfully Transform Them. New York: Currency.

García, O. (2003). Valoración de empresas, gerencia del valor y EVA. Medellín: Digital Express Ltda.

Geroski, P., Machin, S. \& Van Reenen, J. (1993). "The profitability of innovating firms". En: RAND Journal of Economics, 24 (2): 198-211.

Grabowski, H. \& Mueller, D. (1978). "Industrial Research and development, intangible capital stocks, and firm profit rates". En: The Bell Journal of Economics, 9 (2): 328-343.

Green, W. (2011). Econometric analysis. Boston: Prentice Hall.

Hall, J. \& Brummer, M. (1999). The relationship between the market value of a company and internal performance measurements. Recuperado el 3 de enero de 2011, de http://ssrn. com/abstract $=141189$.

House, W., Parks, D. \& Lindstrom, G. (1994). "Relationships between R\&D and profitability: an exploratory comparison 
of two business simulations with two real-world technology intensive industries". En: Developments in Business Simulation \& Experiential Exercises, 21: 75 -78.

Iñiguez, R. \& Poveda, F. (2001). "Medidas de creación de valor para los accionistas: EVA ${ }^{\circledast}$ vs beneficios". En: Revista Española de Financiación y Contabilidad, 107: 207-237.

Koski, H. \& Kretschmer, T. (2010). "New product development and firm value in mobile handset production". En: Information Economics and Policy, 22: 42-50.

Langebaek, A. \& Vasquéz, D. (2007). "Determinantes de la actividad innovadora en la industria colombiana". En Borradores de Economía, 433. Banco de la República. Colombia.

Lehn, K. \& Makhija, A. (1997). "EVA, accounting profits and CEO turnover: An empirical examination, 1985-1994". En: Journal of Applied Corporate Finance, 10 (2): 90-97.

Lovata, L. (2001). "Does market valued added increase with integration of economic value added in performance valuation?" En: Accounting Enquiries, 10 (1): 147-170.

Martin, J. \& Petty, J. (2001). La gestión Basada en el Valor. La respuesta de la empresa a la revolución del accionista. Barcelona: Ediciones Gestión 2000 S.A.

Miller, M. \& Modigliani, F. (1963). "Corporate income taxes and the cost of capital: a correction". En: The American Economic Review, 53: 433 - 443.
Morbey, G. \& Reither, R. (1990). "How R\&D affects sales growth, productivity and profitability". En: Research-Technology Management, 33 (3): 11-14.

O’Byrne, F. (1996). "EVA and market value”. En: Journal of Applied Corporate Finance, 9 (1): 116-125.

ODCE \& Eurostat (2006). Manual de Oslo. Guía para recogida e interpretación de datos sobre innovación (3a. ed.).TRAGSA (Original en inglés y francés, 2005).

Porter, M. (1988).Ventaja competitiva (2a. ed.). México: CECSA. (Original en inglés, 1985).

Pauwels, K., Silva-Risso, J., Srinivasan, S. \& Hanssens, D. (2004). "New products, sales promotions, and firm value: The case of the automobile industry". En: Journal of Marketing, 68: 142-56.

Scott, M. (1999). El proceso de creación de valor en la empresa. Análisis y comentarios. Bilbao: Ediciones Deusto S.A. (original en inglés, 1998).

Sorescu, A., Chandy, R. \& Prabhu, J. (2003). "Sources and financial consequences of radical innovation: Insights from pharmaceuticals". En: Journal of Marketing, 67: 82-101.

Sorescu, A. \& Spanjol, J. (2008). Innovation's effect on firm value and risk: Insights from consumer packaged goods. En: Journal of Marketing, 72 (March): 114-132.

Stewart, B. (2000). En busca del valor. Barcelona: Ediciones Gestión 2000. (Original en inglés, 1991). 